\title{
A Controllable Environmental-Cell (EC) for Wet Environmental TEM (WETEM)
}

\author{
Ryosuke Kagawa $^{1}$, Yuma Kuwamura ${ }^{1}$, Wen-An Chiou $^{2}$ and Hiroki Minoda ${ }^{1}$
}

1. Department of Applied Physics, Tokyo University of Agriculture and Technology, Nakacho, Koganei,Tokyo 184-8588, Japan

2. NISP Lab, NanoCenter, University of Maryland, College Park, MD 20742-2831, USA

Water is an essential constituent of all biological materials as well as many non-biological materials. Not only the removal of water may result in undesirable morphological and structure change, the inability to sustain the hydrated conditions in the microscope also prevents the study of reactions which take place in aqueous environment. Much attention has thus been devoted to the development of wet environmental TEM (WETEM) in recent years due to the unique capability of imaging/investigating wet materials and resolving real-time dynamic environmental processes at micro- and nano-scales. Although a simple and cost-effectively constructed wet environmental-cell (EC) was able to examine materials in hydrated condition [1], a steady state of pressure in the EC cannot be maintained throughout the investigation. This paper presents a newly developed closed EC system in a TEM that allows for dynamic observation by controlling humidity and air circulating during the experiment.

To ensure the EC maintains controllable moisture/humidity, a two-line sample holder for JEOL 2010 TEM was fabricated based on Fukami's wet EC holder which was used in JEOL 2000EX TEM [2]. The new EC system includes a small EC $0.4 \mathrm{~mm}$ in height and $2 \mathrm{~mm}$ in diameter, and has a gas flow controlling system to control the flow rate of air/gas and the humidity of the EC. To ensure the high vacuum of TEM column and to prevent a rise in pressure (i.e., due to the leakage of the flowing gas in the column and eventually to the gun chamber during in-situ observation), the vacuum system was enhanced by adding: (1) a pumping system for controlling gas flow ((1) in Fig. 1); (2) a turbo molecular pump for additional column pumping ((2) in Fig. 1); and (3) a sputter ion pump to enhance gun chamber vacuum and evacuation independently ((3) in Fig. 1). This new gas flow system provides precise control of the humidity or amount of water in the EC, i.e., around the specimen. Smectite, one kind of clay minerals that is expandable in water, collected from different localities was purified to remove organic matter and other impurities from the original soil. Smectite particles were dispersed in deionized water and/or ethanol. A tiny drop of suspension was pipetted onto the EC for in-situ study.

TEM micrograph reveals typical smectite aggregates and well-defined particle outlines when the sample was in dry state (Fig.2a). The blurred images and the diffused SAD patterns of smectite particles from WETEM observations indicate electron scattering by water molecules in the EC and thus in the specimen (Fig. 2b). While the morphological change (image) may not reflect the humidity change of specimen so precisely, electron diffraction patterns clearly reveal the d-spacing change when the concentration of in-take water vapor changed. A series of SAD patterns were taken at different stages while changing humidity in the EC (i.e., water content in the sample) (Fig. 3). These SAD patterns were obtained from the specimens during evacuation of the wet EC. Analysis of SAD patterns obtained from fully hydrated wet state (Fig. 3b) to dehydrated state (Fig. 3d) illustrates lattice contraction of (h k 0). The d-spacing reduction vs. time of evacuation plots clearly show that the $d$-spacing of $a$ and $b$ axes seem to shrink in the same trend as that of in c axis (Fig. 3e). The new EC vacuum system provides controllable and innovative EC environment for dynamic research.

[1] Y. Kuwamura et.al., Microscopy and Microanalysis 19 supplement 2 (2013) p. 490-491.

[2] A. Fukami et al., Proc. $45^{\text {th }}$ EMSA, Baltimore, (1987), p. 142-143. 
[3] Conventional TEM work performed at NISP Lab was partially supported by NSF-MRSEC (DMR 05-20471) and UMD. WETEM was carried out at TUAT.
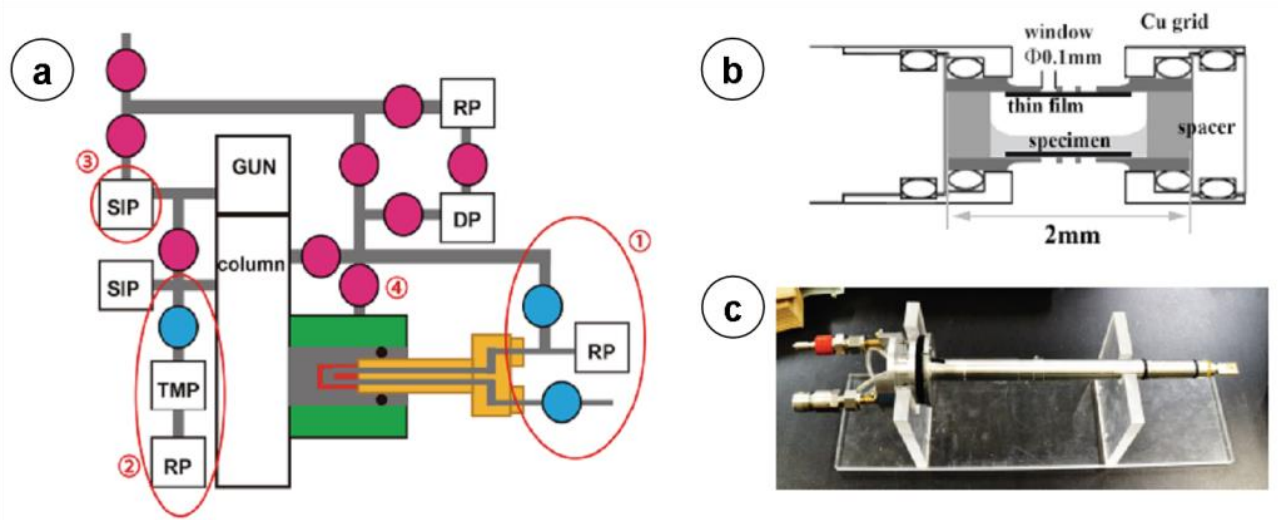

Fig. 1. Schematic diagrams ( $\mathrm{a}$ and $\mathrm{b}$ ) and photograph (c) show the new pressure control system and the new EC TEM holder (JEM2010). Vacuum pumps/controls (1) - (4) were added in the new system.
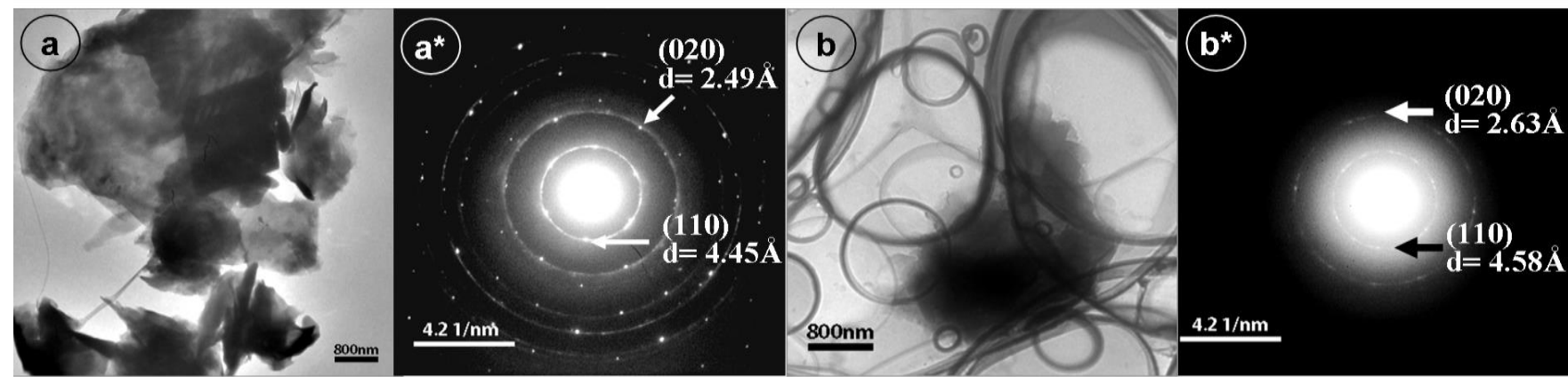

Fig. 2. TEM micrographs and corresponding SAD patterns of smectite clay in dry state (a and $\mathrm{a}^{*}$ ) and in hydrated environment ( $\mathrm{b}$ and $\mathrm{b}^{*}$ ). Note the blurred image and diffused SAD pattern of wet clay.
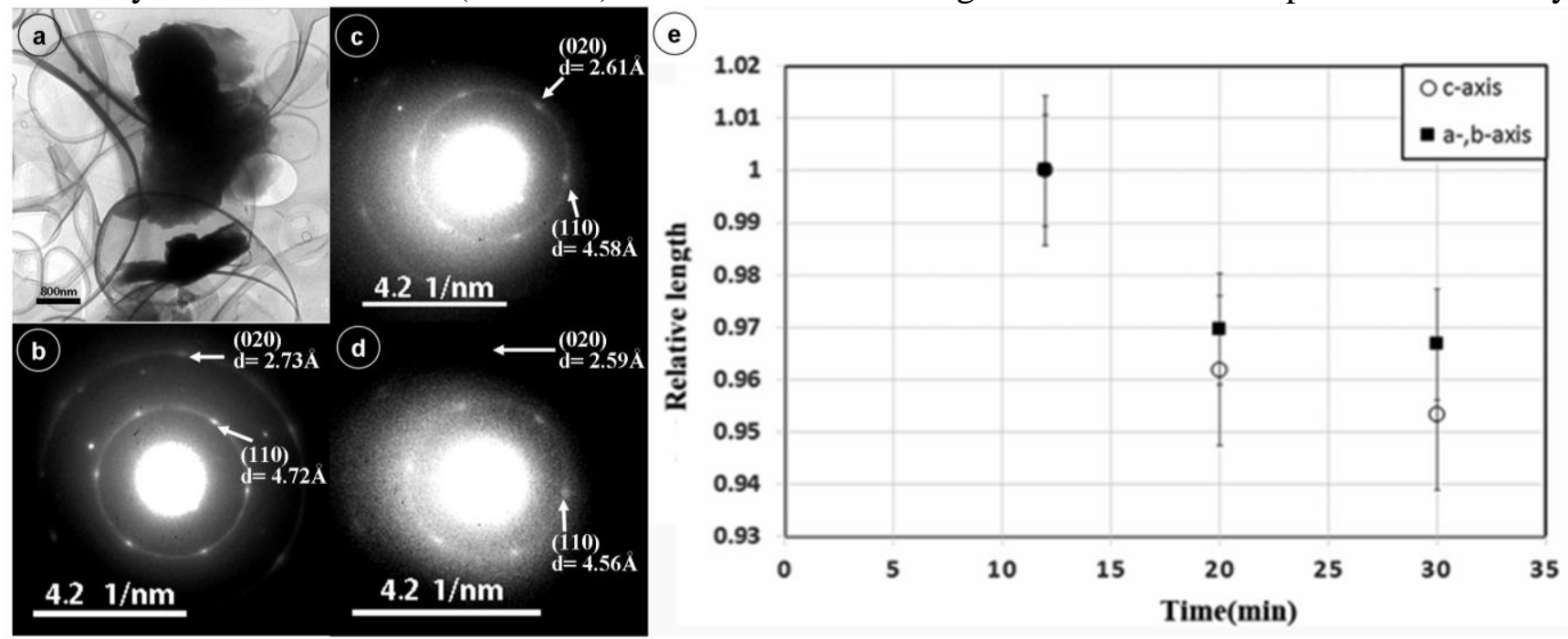

Fig. 3. In-situ WETEM observation shows the change of d-spacing of the same clay particle (a) in different humidity during evacuation of the EC (i.e., dehydration, from (b) to (d)). The relative length (d-spacing) of crystal axes vs. evacuation time of the EC is plotted (e). Through the time the $\mathrm{d}$-spacing change of $\mathrm{a}$ and $\mathrm{b}$ axes is coincided with that of $\mathrm{c}$ axis. The reduction of $\mathrm{d}$-spacing was resulted from the loss of water molecular between tetrahedron and octahedron layers of clay. 